\title{
Closing Remarks at Spin 2018 Symposium
}

\author{
Haiyan Gao*t \\ Department of Physics, Duke University, Durham, North Carolina 27708, USA \\ Triangle Universities Nuclear Laboratory, Durham, NC 27708, USA \\ Duke Kunshan University, Kunshan, Jiangsu 215316, China \\ E-mail: gao@phy.duke.edu
}

In this closing talk, I offer some personal reflection in the context of spin symposia on its global nature, its impact and also on the diverse spin related topics in various sub-fields of physics from some of the most recent symposia. Since the last Spin Symposium in 2016, there have been major developments worldwide on electron-ion colliders, which I also discuss briefly. Finally it is my pleasure to unveil where the next Spin Physics Symposium (Spin 2020) will take place.

23rd International Spin Physics Symposium - SPIN2018 -

10-14 September, 2018

Ferrara, Italy

\footnotetext{
* Speaker.

${ }^{\dagger}$ This work is supported in part by the U.S. Department of Energy, Office of Science, Office of Nuclear Physics under contract No. DE-FG02-03ER41231.
} 
The 23rd spin physics symposium is coming to an end. This has been a very special week - physicists from around the world gathered at the beautiful and historic city of Ferrara, Italy to share the latest discoveries and developments in spin physics and related areas. On behalf of the International Spin Physics Committee (INPC), I like to express our gratitude to our local organizers, especially the chair of the symposium, Dr. Paolo Lenisa for doing such a fabulous job in organizing this wonderful symposium. I also like to thank all the speakers, conveners and participants for making this symposium a great success.

I would like to take this opportunity to share some personal reflection on being here at this physics meeting in Ferrara coming directly from Kunshan, China where I have been devoting a significant amount of my time working on building a new joint venture university, Duke Kunshan University between Duke University in the United States, and Wuhan University in China in the last 4 years or so. University of Ferrara, among the oldest universities in the world was established in 1391. For someone coming from a university that was established in 2014, I am truly humbled and also amazed by how global universities are becoming in the 21 st century. As physicists we are proud of our tradition of addressing some of the nature's most fundamental questions in a global way by working in international collaborations. CERN certainly is one of the most successful examples for major international collaborations in Physics. Spin always has a special place in the development of modern physics. The more recent development of spin physics in nuclear and particle physics benefited enormously from the original polarized deep inelastic scattering measurements carried out by the European Muon Collaboration (EMC) at CERN [1,2]. With the latest development on electron-ion colliders, which I will discuss later, new international collaborations are being formed to uncover the proton spin puzzle and to discover possibly new gluonic matter.

We are also very proud to note that how much more global our spin physics community is becoming in the 21st century. This series of symposium started in 1974 at Argonne National Lab in USA, and has been held in the United States for eight times since its inception. Since 1980, the spin physics symposium started to be held outside the US, and the first such symposium was in Lausanne, Switzerland. In the last forty plus years, it has been held in eight different countries in North America, Europe and Asia with the latest addition of China in 2014. The Beijing Spin 2014 symposium attracted physicists from around the world and also from many parts of China. What was particularly successful with Spin 2014 was the high participation of Chinese graduate students and other young physicists, which was very helpful to the effort of building up a hadronic physics community in China. This symposium also has made a huge impact on the hadronic physics community in China in its pursuit of a polarized electron-ion collider, which has been proposed for construction in China (more later). Going into the future, we hope this series of symposium will become even more global by adding more countries and more continents to the list of countries and continents which have hosted a spin physics symposium.

It has been more than 30 years since the EMC Collaboration released its experimental finding, which precipitated the original "Proton Spin Crisis" [1, 2]. The "Proton Spin Crisis" referred to the original EMC discovery that the spin of quarks inside the proton almost contributed nothing to the proton spin, which was completely opposite to the picture provided by the naive quark model. So this finding was termed as the "Proton Spin Crisis", which we now more accurately characterize it as the proton spin puzzle. The original EMC proton spin result motivated both experimental and theoretical activities in the last 3 decades worldwide in order to fully understand where the proton 
spin comes from. We now know after more precise experimental results and theoretical studies, especially advances in the lattice QCD calculations that the spin of quarks inside the proton contributes about a quarter to its spin, and the rest comes from the spin of the gluons and orbital angular momenta of quarks and gluons. While major progresses have been made in understanding where the proton spin comes from especially from polarized inclusive and semi-inclusive deep inelastic scattering experiments and polarized proton-proton scattering experiments, the full resolution of the proton spin puzzle requires a next generation and an ultimate QCD machine, such as the proposed U.S. based electron-ion collider (EIC). The concept and the physics goals for a US based polarized EIC have been in the making by the US QCD nuclear physics community and its accelerator counterpart for more than two decades. In the 2015 Long Range Plan (LRP) for Nuclear Science, "a high-energy high-luminosity polarized EIC as the highest priority for new facility construction following the completion of FRIB" was one of the recommendations [3]. Following the 2015 LRP, the US Department of Energy asked the US National Academies of Sciences \& Engineering \& Medicine to conduct an assessment of U.S.-Based Electron-Ion Collider Science in the fall of 2016. In July 2018, the US National Academies of Sciences \& Engineering \& Medicine released an assessment committee report on U.S.-Based Electron-Ion Collider Science [4]. The assessment committee highlighted three important science questions that the U.S. based EIC can uniquely address. These are: How does the mass of the nucleon arise? How does the spin of the nucleon arise?, and What are the emergent properties of dense systems of gluons? The proton spin is one of the centerpieces of the core science of the proposed EIC facility.

There are two parallel efforts in the United States in the design of a polarized EIC, with one based at the Brookhaven National Lab (BNL), known as eRHIC [5]. The other one, known as JLEIC, is based at the Thomas Jefferson National Accelerator Facility (Jefferson Lab) [6]. Fig. 1 shows the schematic layout of eRHIC, and Fig. 2 shows that of JLEIC. The eRHIC design takes advantages of the existing infrastructure at BNL, such as the alternating gradient synchrotron (AGS), and the Relativistic Heavy Ion Collider (RHIC). The JLEIC design utilizes the existing energy upgraded $12-\mathrm{GeV}$ electron facility, and the central part of the design is two shape- 8 collider rings with one for electrons and one for ions. Both designs will allow for a range of center-of-mass energies and polarized luminosities with the eRHIC design offering somewhat higher energies and the JLEIC higher polarized luminosities.

In addition to the proposed U.S. based EIC, there are two major efforts with one in China and another in Europe. The Large Hadron electron Collider (LHeC) at CERN [7] has been proposed for a center-of-mass energy higher than $1 \mathrm{TeV}$ with physics goals of Higgs physics, searches for new phenomena and high precision electroweak and QCD physics, which are somewhat different from those of the U.S. based EIC. There has been a major push by the Chinese hadronic physics community to construct an Electron-Ion-Collider in China (EicC) led by the Institute of Modern Physics (IMP) of the Chinese Academy of Sciences [8]. Currently, IMP is leading the effort in the construction of the High Intensity Heavy-Ion Accelerator Facility (HIAF) in the southeast part of China. The proposed EicC has two stages with EicC-I as an extension to the aforementioned HIAF with polarized electrons of $3-5 \mathrm{GeV}$ on $12-23 \mathrm{GeV}$ polarized protons. This proposed EicC-I will be complementary to the proposed U.S. based EIC and also complementary to ongoing and future fixed target experiments in US, Europe and elsewhere. EicC-II will include polarized electrons up to $10 \mathrm{GeV}$ on $60-100 \mathrm{GeV}$ polarized protons. Fig. 3 shows the schematics of the proposed EicC-I 


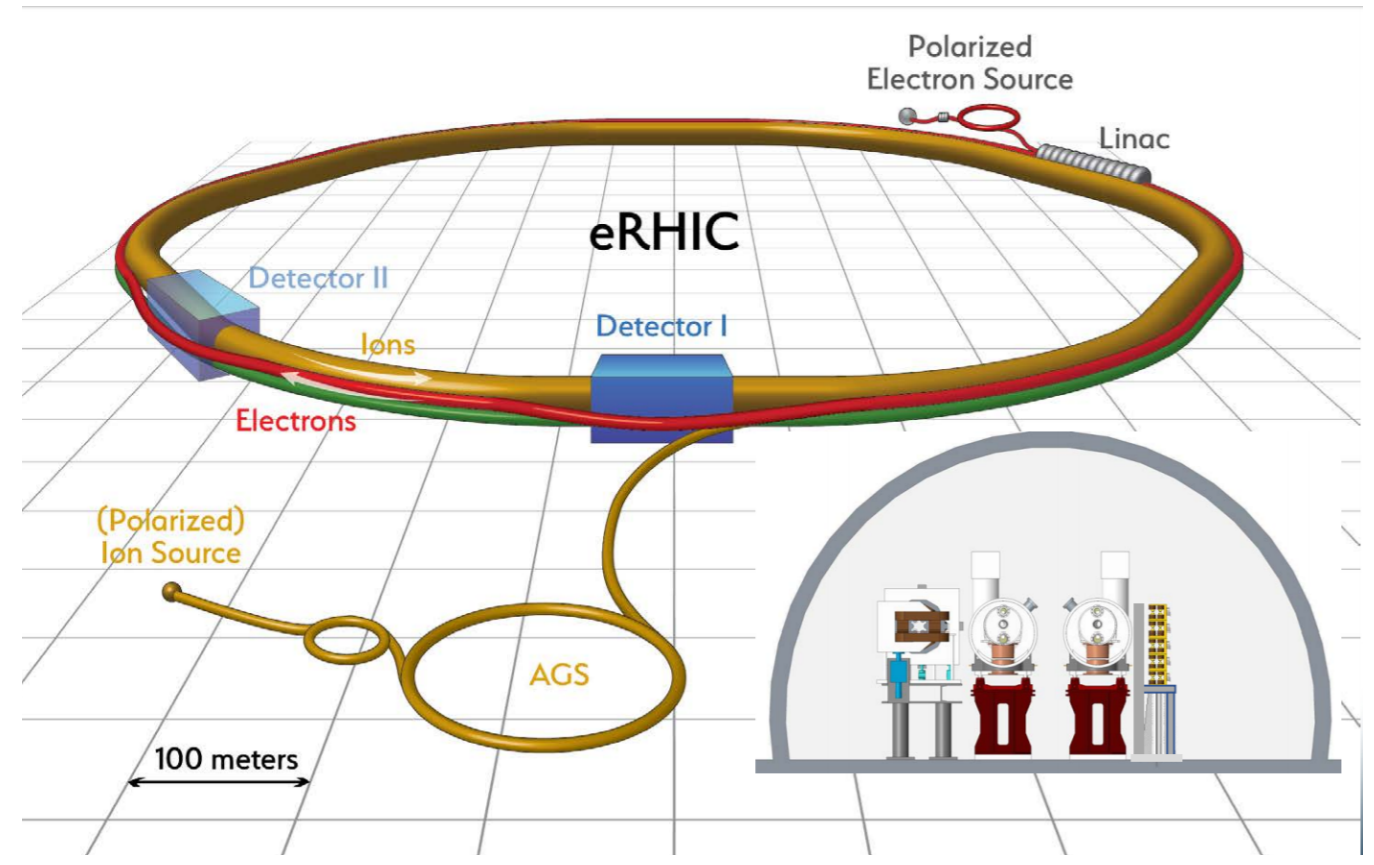

Figure 1: Schematic layout of eRHIC, showing the existing hadron injector complex (ion source, alternating gradient synchrotron (AGS), etc.) and Relativistic Heavy Ion Collider (RHIC) tunnel containing two superconducting hadron rings. The addition of a polarized electron source, full-energy injector linac and high-energy electron ring in the same RHIC tunnel opens up the possibility of polarized electron-hadron collisions. SOURCE: C. Montag et al., 2017, "Overview of the eRHIC Ring-Ring Design," IPAC 2017, and a report of the National Academies of Science, Engineering and Medicine, 2018, "An Assessment of U.S.-Based Electron-Ion Collider Science,” Washington DC, the National Academies Press.

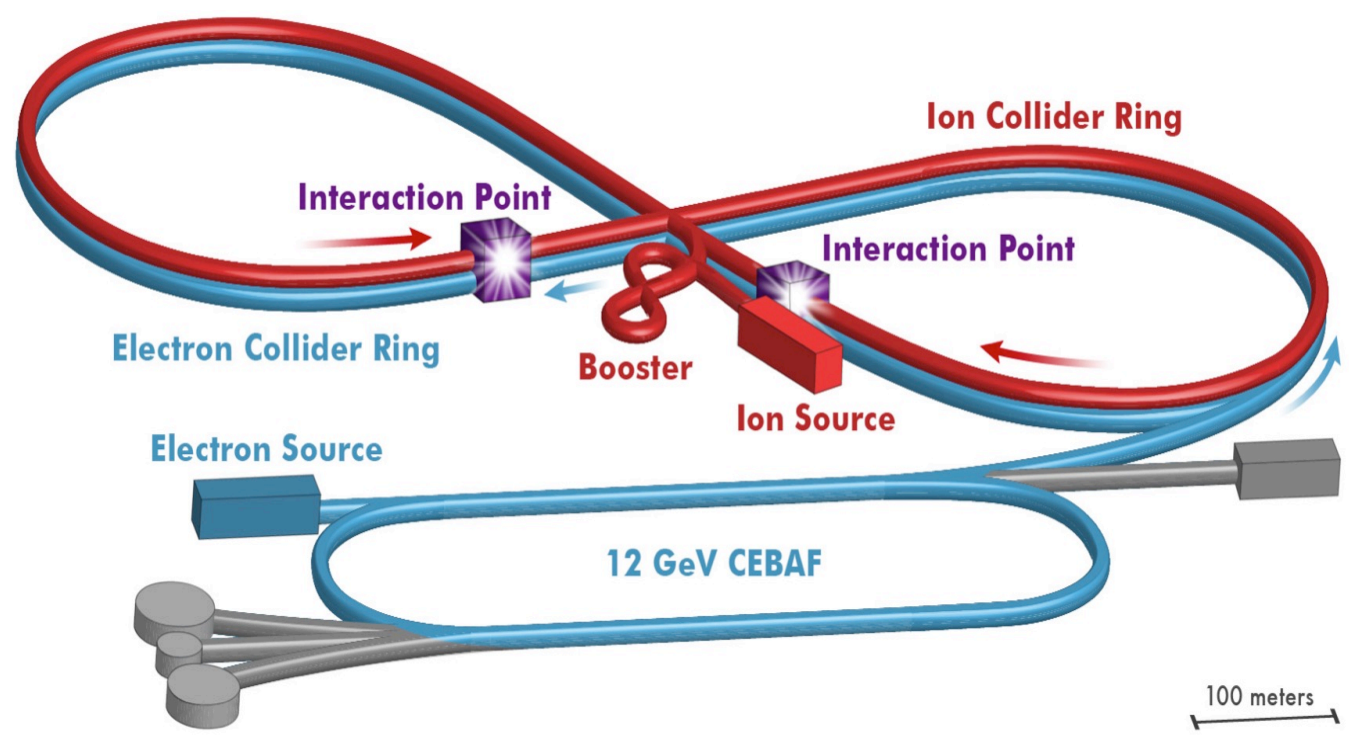

Figure 2: Schematic layout of the Jefferson Laboratory Electron Ion Collider design. SOURCE: Thomas Jefferson National Accelerator Facility, and a report of the National Academies of Science, Engineering and Medicine, 2018, “An Assessment of U.S.-Based Electron-Ion Collider Science," Washington DC, the National Academies Press. 
and EicC-II. Fig. 4 shows luminosities versus center-of-mass energies for a number of fixed target experiments using lepton beams, and also existing and proposed electron/positron-ion colliders in the world. The next a few years will be very exciting as more developments on these EIC efforts will take place and in US a down selection process will likely take place in the near future.

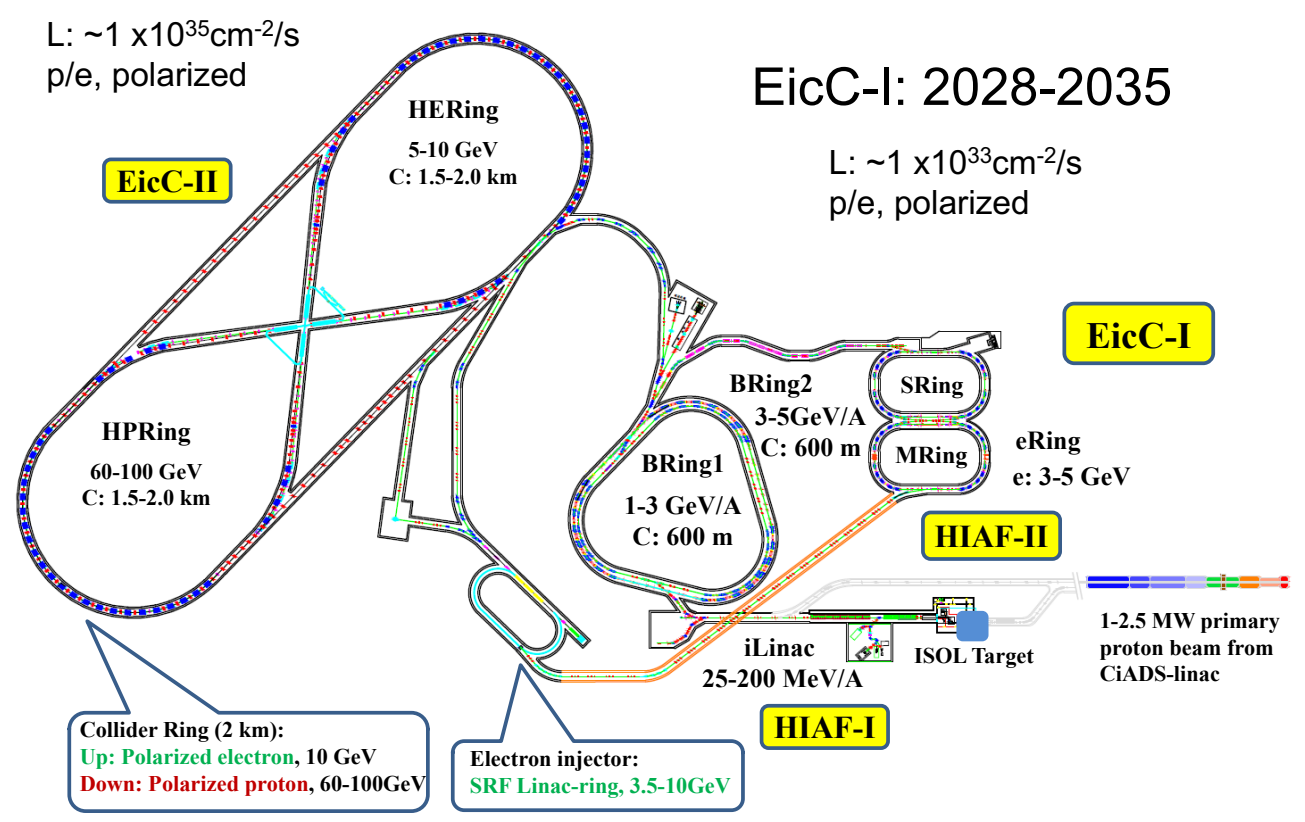

Figure 3: Schematic layout of the EicC conceptual design. SOURCE: Jianchen Yang.

Spin 2018 is timely and at this symposium we heard talks covering the latest results concerning the proton spin puzzle, and also talks on both the U.S. based EIC and also EicC. In more recent years the spin physics related topics covered at the symposia have also been more diverse. At the Spin 2018 symposium, topics included spin and cosmology, quantum technologies on manipulating individual spins, hyperpolarizing ${ }^{13} \mathrm{C}$ spins by dynamic nuclear polarization for MRI applications, as well as applications to other fields in addition to spin physics and phenomena in atomic, nuclear and particle physics. Previously at the Spin 2014 symposium in Beijing, in addition to a plenary talk on medical imaging with highly spin polarized molecules and a public lecture on quantum anomalous hall effect and information technology, there were parallel sessions on spins in condensed matter physics, quantum information and computation. Having such diverse spin related topics at spin symposia is healthy for our field as physicists can learn from each other and new ideas and techniques will come out of "collisions" of people from different sub-fields of physics.

Before Spin 2018, the most recent spin symposium that took place in Italy was Spin 2004 in Trieste. So it has been fourteen years for the symposium to return to Italy. Interestingly, it will also take fourteen years for this symposium to return to Japan - Spin 2020 will take place in Matsue, Japan in September, 2020 and the most recent spin symposium in Japan was Spin 2006 in Kyoto, Japan. Matsue is a famous and interesting area for 2000 years of Japanese history from creation myth era to the present time. Spin 2020 will be hosted by RIKEN and we look forward to another exciting spin symposium in 2020 ! 


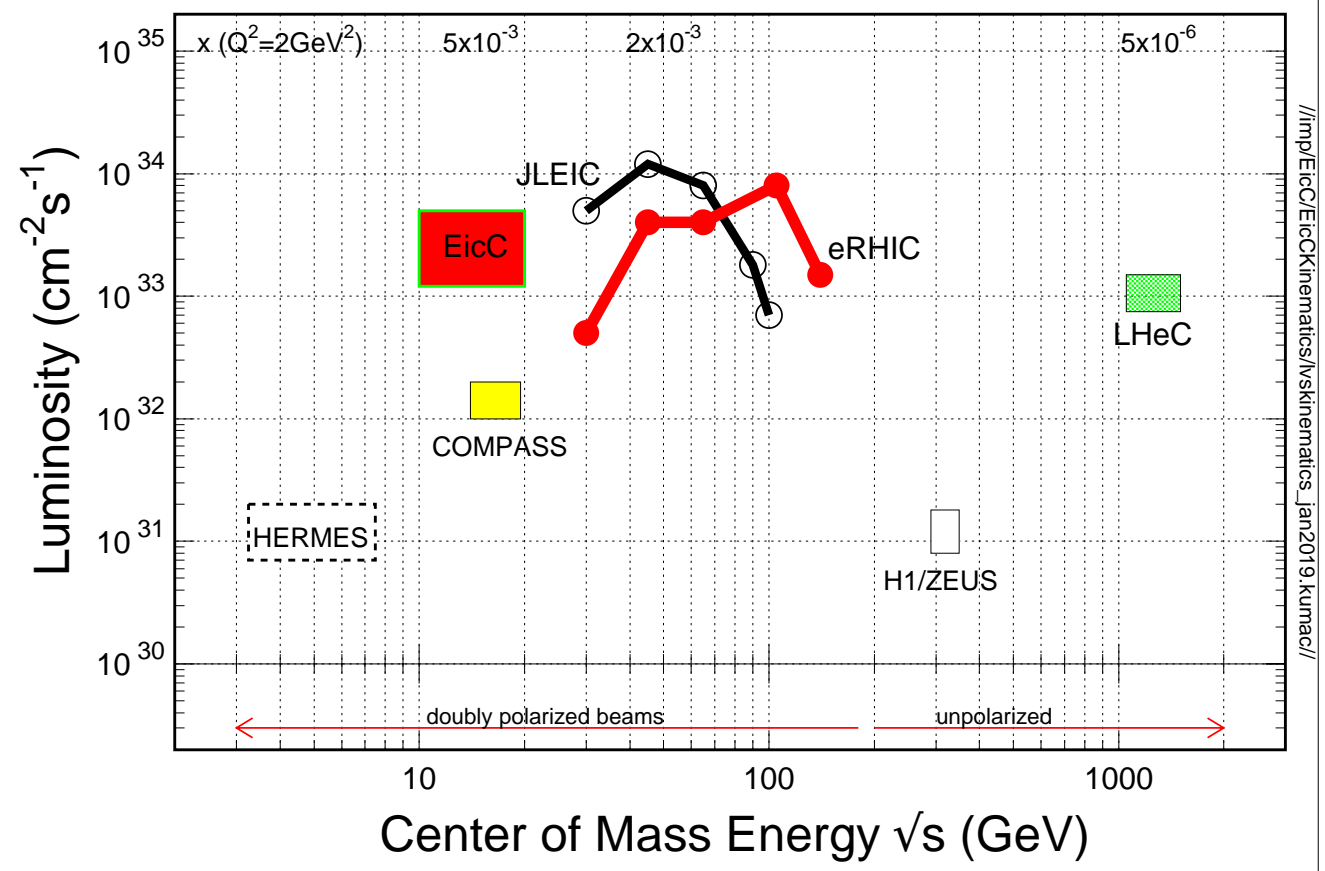

Figure 4: Comparison on luminosities and center of mass energies of the experiments. The HERA with $\mathrm{H} 1$ and ZEUS and the proposed $\mathrm{LHeC}$ are unpolarized electron/positron ion colliders. The HERMES and COMPASS are fixed target experiments with polarized beams and targets. The proposed JLEIC, eRHIC and EicC are electron ion colliders with doubly polarized beams. SOURCE: Xurong Chen

\section{Acknowledgments}

I like to thank Dr. $\mathrm{Nu} \mathrm{Xu}$ for his help in providing the materials related to EicC, and Drs. Tianbo Liu and Zhiwen Zhao for their help with the manuscript.

\section{References}

[1] J. Ashman et al. (European Muon Collaboration), Phys. Lett. B 206, 364 (1988).

[2] J. Ashman et al. (European Muon Collaboration), Nucl. Phys. B 328, 1 (1989).

[3] The 2015 Long Range Plan for Nuclear Science.

[4] A report of the National Academies of Science, Engineering and Medicine, "An Assessment of U.S.-Based Electron-Ion Collider Science," Washington DC, the National Academies Press, 2018.

[5] E. C. Aschenauer et al., arXiv:1409.1633 [physics.acc-ph].

[6] S. Abeyratne et al., arXiv:1209.0757 [physics.acc-ph].

[7] J. L. Abelleira Fernandez et al., J. Phys. G 39, 075001 (2012).

[8] X. Chen, PoS DIS 2018, 170 (2018). 


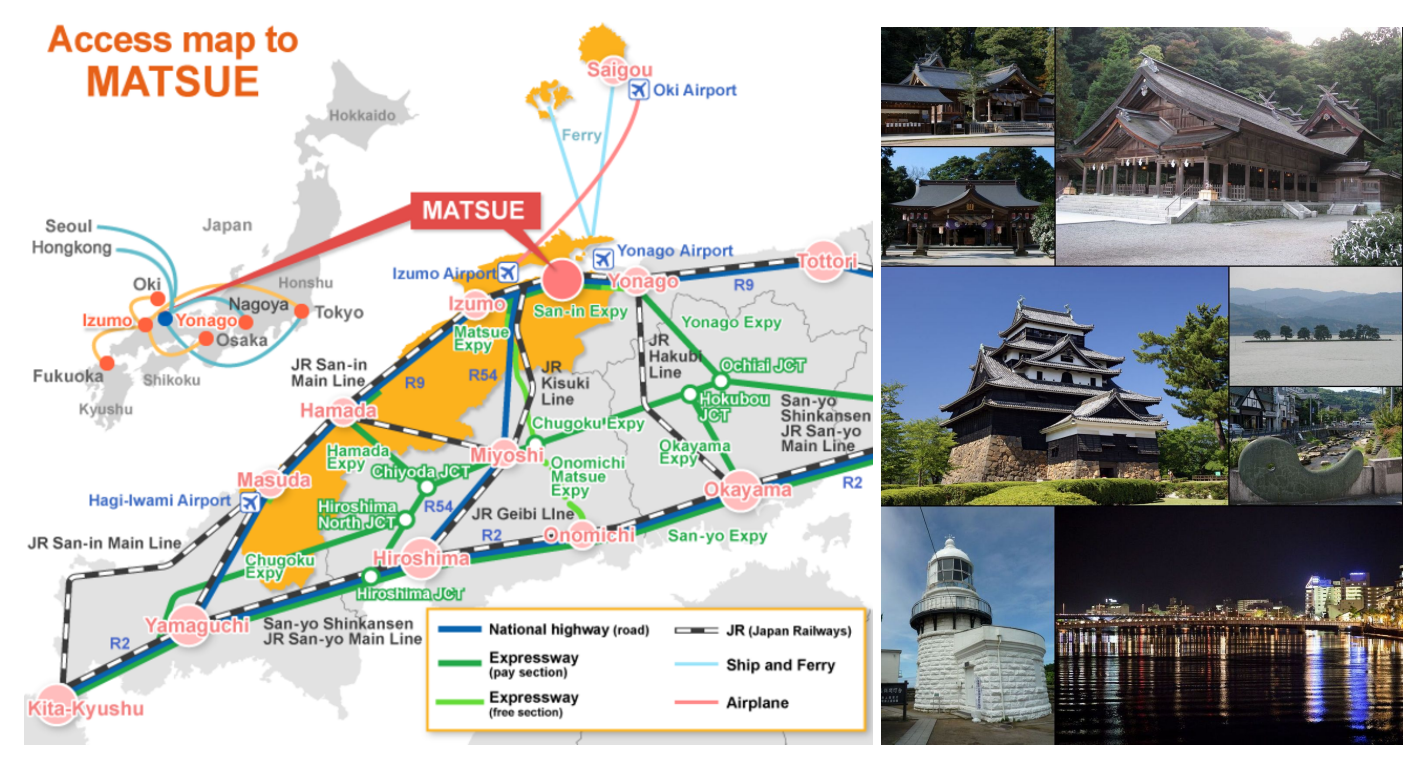

Figure 5: Spin 2020 in Matsue, Japan, September, 2020. 